\title{
Overexpression of miR-140-5p inhibits lipopolysaccharide- induced human intervertebral disc inflammation and degeneration by downregulating toll-like receptor 4
}

\author{
QIANG ZHANG ${ }^{*}$, YIPING WENG ${ }^{*}$, YUQING JIANG, SHUJIE ZHAO, DONG ZHOU and NANWEI XU \\ Department of Orthopaedics, The Affiliated Changzhou No. 2 People's Hospital with \\ Nanjing Medical University, Changzhou, Jiangsu 213000, P.R. China
}

Received September 15, 2017; Accepted April 20, 2018

DOI: $10.3892 /$ or.2018.6488

\begin{abstract}
Toll-like receptor 4 (TLR4) families are receptors for ligands that initiate extracellular or intracellular signaling, such as lipopolysaccharides (LPS). It has been reported that TLR4 activation resulted in the upregulation of a coordinated set of proinflammatory mediators and inhibition of matrix expression in the intervertebral disc (IVD). miR-140-5p (miR140) is reported to participate in cellular anti-inflammatory processes and target TLR4. In the present study, we investigated the relationship between TLR4 and miR-140 in IVD degeneration. The expression of TLR4, interleukin (IL)-6, IL-I, L-1 $\beta$ and tumor necrosis factor (TNF)- $\alpha$ was higher, in high-grade IVD degeneration tissues than in low-grade tissues. In contrast, the expression of miR-140, aggrecan and collagen type II was lower in high-grade IVD degeneration tissues than in low-grade IVD degeneration tissues. LPS stimulation resulted in significant increases in TLR4 expression and decreases in miR-140 expression in nucleus pulposus (NP) cells and TLR4 was identified as a target of miR-140 by dual-luciferase reporter assay. The overexpression of miR-140 inhibited the upregulation of the expression of TLR4, TNF- $\alpha$, IL-1 $\beta$ and IL- 6 inflammation cytokines, and the activation of $\mathrm{NF}-\kappa \mathrm{B}$ and reversed the downregulation of the expression of aggrecan and collagen type II induced by LPS stimulation. In conclusion, the present study may lead to a greater understanding of IVD degeneration and provide new insights into the treatment of this disease.
\end{abstract}

Correspondence to: Professor Dong Zhou or Professor Nanwei Xu, Department of Orthopaedics, The Affiliated Changzhou No. 2 People's Hospital with Nanjing Medical University, 29 Xinglongxiang Street, Changzhou, Jiangsu 213000, P.R. China

E-mail: zhoudong1012@hotmail.com

E-mail: nwxu_no2@163.com

*Contributed equally

Key words: intervertebral disc degeneration, inflammation, LPS, TLR4, miR-140-5p, ECM

\section{Introduction}

Degeneration of the intervertebral disc (IVD) is a pathological process characterized by changes in architecture and biochemical composition, which alters the ability of the IVD to bear load and can lead to disk herniation (1-5). IVDs have a gelatinous center, known as the nucleus pulposus (NP), encompassed by coaxial lamellae that form the inner and outer annulus fibrosus. Degeneration of the IVD can cause metabolic changes in the extracellular matrix (ECM) leading to reduced water content and a loss of the boundary between the outer annulus fibrosus and the NP (6-8). The ECM is predominantly composed of collagen type II and proteoglycans, mainly in the form of aggrecan $(9,10)$. Aggrecan occurs in the NP and in the inner annulus, where it is associated with cartilage differentiation (10). Studies using tissue isolated from surgical patients have revealed that ECM degeneration is associated with loss of proteoglycan content (11). Spinal degenerative diseases are characterized by bone marrow and endplate lesions that are visible on magnetic resonance imaging (12). These degenerative alterations in the vertebral end plates are known as modic changes and are considered to contribute to pain development $(13,14)$. Modic changes are not only related to traumatic injury, bacterial infection and genetics but are also associated with localized inflammation, since proinflammatory crosstalk between the bone marrow and IVDs has recently been identified (14-16).

Proinflammatory cytokines are reported to play an important role in ECM breakdown in relation to IVD degeneration (17,18). Levels of interleukin (IL)-1 $\beta$, IL-6, IL-10 and tumor necrosis factor (TNF)- $\alpha$ have been found to be significantly elevated in patients with IVD degeneration (19) and played an important role in IVD degeneration (20). IL-1 $\beta$ is a predominate cytokine that is upregulated in degenerating IVDs and is considered to be a principal mediator in the breakdown of ECM (21). TNF- $\alpha$ can enhance matrix-degrading enzyme activity and increase NP cell apoptosis (17,22,23). Li et al also reported that TNF- $\alpha$ induced NP cell inflammation and apoptosis by activating $\mathrm{NF}-\kappa \mathrm{B}$ signalling (24). Heme oxygenase-1 (HO-1) has also been reported to participate in the cellular anti-inflammatory processes relating to IVD degeneration (25). When HO-1 is induced, the effects of IL-1 $\beta$ on the 
expression of the catabolic markers matrix metalloproteinases (MMP)-1, 3, 9 and 13 are reversed (25).

Toll-like receptor (TLR) signaling pathways were also reported to increase the level of proinflammatory cytokines, TNF-a, IL-1, IL-6 and IL-8, leading to inflammation and pain $(26,27)$. The functions of the toll-like receptor 4 (TLR4) proteins included the activation of the innate immune system (28). In addition, TLR4 was highly expressed in cartilage with advanced OA and played a key role in cartilage degradation $(29,30)$. IVD cells have been found to express TLR4 and respond to TLR4 activation induced by lipopolysaccharide (LPS) treatment by upregulating a coordinated set of inflammatory cytokines and inhibiting ECM expression in IVD in vitro and in vivo (31). Ligand binding to TLRs initiated a signaling cascade that led to the activation of nuclear factor (NF)- $\kappa \mathrm{B}$ and MAPKs, which promoted the production of inflammatory cytokines, chemokines and degradative enzymes (32). NF- $\kappa \mathrm{B}$ is an heterodimer consisting of p65 and p50 subunits and is sequestered in the cytoplasm by inhibitor proteins, the IкBs (33). TLR4 is known to recognize LPS, a component found in bacterial cells (34). As a TLR ligand, LPS can decrease proteoglycan (PG) synthesis, aggrecan production, and the expression of collagen II by TLR4 signaling in both murine and human articular chondrocytes (30). IL-1 and LPS-mediated NP cell inflammation and PG and matrix-degrading enzyme production were antagonized by LfcinB treatment (35). LPS can induce TLR signalling in intervertebral disc cells, leading to increased expression of proinflammatory cytokines (36). LPS-induced aggrecan and collagen II downregulation was inhibited by carthamin yellow through the suppression of the MAPK pathway activation (37). LPS stimulates NF- $\mathrm{B}$ binding in the TLR4 gene promoter, whereas TLR4 expression is blocked by $\mathrm{NF}-\kappa \mathrm{B}$ inhibitors $(31,38)$. Furthermore, LPS was reported to induce inflammation in acute kidney injury (AKI) by activating the TLR4/NF- $\mathrm{B}$ signaling pathway (39).

MicroRNAs (miRNAs) are small (18-24 nucleotides long) non-protein-coding RNAs that regulate gene expression at the post-transcriptional level (40). Microarray expression analyses have revealed a significant miRNA dysregulation in osteoarthritis which indicated that miRNAs may be involved in the pathology of degenerative joint diseases (41). miR-140 is known to be a cartilage-specific miRNA, with a major role in pathogenesis $(42,43)$. In a recent study, miR-140 was found to protect chondrocytes against the anti-proliferation and cell-matrix signaling changes induced by cytokine IL-1 $\beta$ in an osteoarthritis model (44). Karlsen et al (45) reported that overexpressing miR-140 can rescue IL-1 $\beta$-suppressed aggrecan and the expression of SOX9 and inhibit inflammation in OA. In a recent study, miR-140 was found to bind to the 3'-UTR of SIRT1, a member of the sirtuin family of proteins, in a dual-luciferase reporter assay performed in 293 cell lines (46). SIRT1 has been shown to deacetylate and thereby, deactivate the p53 protein (47), which is an essential metabolic regulatory transcription factor (48). Li et al (49) reported that miR-140-5p inhibited cell proliferation and inflammatory cytokine secretion by downregulating TLR4 in synovial fibroblasts. In a previous study using microarray analysis, we revealed that miR-140 was more upregulated in articular chondrocytes than in mesenchymal stem cells and modulated IL-1 response (50).
We have also discovered that miR-140 targeted the TLR4 3'-UTR using TargetScan. In the present study, we assessed the miRNA-140 3'-UTR binding site of TLR4 by a dual-luciferase reporter assay performed in NP cells. TLR4, inflammation cytokines, aggrecan and collagen type II expression were detected by qRT-PCR induced by LPS stimulation in vitro. TLR4 is a major receptor of LPS. We also assessed the impact of miR-140 on proinflammatory cytokine levels, aggrecan and collagen type II expression in relation to TLR4 expression induced by LPS stimulation. Therefore, we assessed whether miR-140 could prevent the progression of inflammation and degeneration in LPS-induced NP cells by inhibiting the expression of TLR4.

\section{Materials and methods}

Human tissue samples. The Research Ethics Committee of Changzhou No. 2 People's Hospital (Changzhou, China) approved the resection of all specimens. Written informed consent was obtained by the patients or their relatives to obtain human intervertebral tissue at surgery. NP samples $(n=22)$ were obtained from patients (33-78 years old; mean age, 53 years) who underwent disc resection surgery or spinal fusion to relieve lower back pain. In addition the NP specimens were grouped according to a grading system for IVD degeneration, which was based on preoperative magnetic resonance images (MRI) (51). No medications or anti-inflammatory drugs were used before surgery. In the present study, samples graded II-III $(n=12)$ were designated the low degeneration group (used as a relatively normal control); samples graded IV-V $(n=10)$ were designated the high degeneration group. All tissues were snap-frozen in liquid nitrogen at the time of surgical removal and stored at $80^{\circ} \mathrm{C}$ or were fixed in $10 \%$ neutral buffered formalin until use.

Isolation of human NP cells. NP tissues were identified by their macroscopic morphology and were carefully separated from any obvious granulation tissue, cartilaginous endplates, or annulus fibrosus as previously described (52). Isolation of NP cells was performed as previously described (25). Briefly, cells were extracted from minced NP tissue specimens from 12 low graded II-III, who underwent posterior discectomy surgery of lumbar degenerative disease, by digesting in $2 \mathrm{U} / \mathrm{ml}$ protease in Dulbecco's modified Eagle's medium (DMEM)/F12 (Gibco; Thermo Fisher Scientific, Waltham, MA, USA) for $30 \mathrm{~min}$ at $37^{\circ} \mathrm{C}$ followed by $0.25 \mathrm{mg} / \mathrm{ml}$ type II collagenase (Gibco; Thermo Fisher Scientific) for $4 \mathrm{~h}$ at $37^{\circ} \mathrm{C}$. The cell suspension was centrifuged at $800 \mathrm{x}$ g for $5 \mathrm{~min}$ and NP cells were resuspended in DMEM/F12 (10\% FBS, $100 \mathrm{U} / \mathrm{ml}$ penicillin, $100 \mu \mathrm{g} / \mathrm{ml}$ streptomycin and $1 \% \mathrm{~L}$-glutamine). Subsequently, the NP cells were treated with $10 \mu \mathrm{g} / \mathrm{ml}$ of LPS (Sigma-Aldrich; Merck KGaA, Darmstadt, Germany).

Cell transfection. For miR-140 overexpression and knockdown, miR-140 mimics, miR-140 inhibitor and two scrambled miRNAs used as negative controls (mimics NC for miR-140 mimics and inhibitor-NC for miR-140 inhibitor, respectively), were purchased from Shanghai GeneChem, Co., Ltd. (Shanghai, China). The $20 \mathrm{nM}$ miRNAs were transfected into NP cells using Lipofectamine 2000 (Invitrogen; Thermo Fisher Scientific) following the manufacturer's instructions 
$q R T-P C R$. Total RNA was extracted from cultured NP cells and degenerative IVD tissues using TRIzol reagent (Invitrogen; Thermo Fisher Scientific) following the manufacturer's instructions. RNA molecules smaller than 200 nucleotides in size were purified using a mirVana miRNA isolation kit (Ambion, Austin, TX, USA) according to the manufacturer's instructions, as previously described (53). Complementary DNA (cDNA) was synthesized using an M-MLV kit (Life Technologies; Thermo Fisher Scientific). qRT-PCR was carried out using the Platinum SYBR-Green qPCR SuperMix-UDG kit (Life Technologies; Thermo Fisher Scientific) on an ABI Prism 7500 PCR system (Applied Biosystems; Thermo Fisher Scientific). Data were normalized to $\beta$-actin. qRT-PCR analysis of mature miR-140 was performed using a TaqMan MicroRNA Assay kit (Applied Biosystems; Thermo Fisher Scientific). Briefly, TaqMan-based real-time quantification of miR-140 included two steps: stem-loop RT and real-time PCR. Stem-loop RT primers bound at the 3 ' portion of miR-140 molecules and were reverse transcribed with reverse transcriptase. Subsequenlty, the RT product was quantified using conventional TaqMan PCR that included miR-140-specific forward primer, reverse primer and a dye-labeled TaqMan probe. The purpose of the tailed forward primer at the 5' end was to increase the melting temperature (Tm) of the miR-140 molecules. Data were normalized to U6 snRNA. All primers are listed in Table I. Each test was performed in triplicate. Relative expression was calculated using the $2^{-\Delta \Delta \mathrm{Ct}}$ method (54).

Western blot analysis. Total protein was extracted from NP cells using a protein extraction kit and quantified using a BCA assay kit (Sigma-Aldrich; Merck KGaA). Following separation by SDS-PAGE, $60 \mu \mathrm{g}$ of protein was transferred to a PVDF membrane and blocked for $1 \mathrm{~h}$ with TBS buffer (10 mM Tris- $\mathrm{HCl}, \mathrm{pH} 7.5$ and $150 \mathrm{mM} \mathrm{NaCl}$ ) containing 5\% skimmed milk. The membrane was then probed with primary antibody at $4^{\circ} \mathrm{C}$ overnight. The primary antibodies were TLR4 (1:500; cat. no. 13556; Abcam) and IאB $\alpha$ (1:500; cat. no. 4814), p-IкB $\alpha$ (1:500; cat. no. 9246), p65 (1:500; cat. no. 8242), and p-p65 (1:500; cat. no. 3039) (all were from Cell Signaling Technology, Beverly, MA, USA). Following three 5-min washes in TBS containing Tween-20 (TBST), the membrane was probed with secondary antibody, goat anti-rabbit IgG-HRP (1:10,000; cat. no. ab6721) and rabbit anti-mouse IgG H\&L (HRP) (1:10,000; cat. no. ab6728) (all from Abcam) at room temperature for $2 \mathrm{~h}$. After further washing in TBST, immunolabeling was detected using Pierce ECL Western Blotting Substrate (Pierce Biotechnology; Thermo Fisher Scientifc). $\beta$-actin was included as a control and densitometric analysis was performed to determine the relative expression of the target proteins.

Luciferase reporter assay. The luciferase reporter assay was performed as previously described (39). The putative miR-140 binding sites in the 3'-UTR of TLR4 was predicted using TargetScan (http://www.targetscan.org); 3'-UTR cDNA fragments of TLR4 containing the putative wild-type or mutant miR-140 binding sites were amplified using the following primers: wild-type 3'-UTR of TLR4, forward, 5'-GTTTAAG ACGTGCTTCAAATATCCA-3' and reverse, 5'-TGATAAG ACCAGGAAGCGGA-3'; mutant 3'-UTR of TLR4, forward,
Table I. The primer sequences.

\begin{tabular}{ll}
\hline Gene & \multicolumn{1}{c}{ Primer sequence } \\
\hline TLR4 & F: 5'-TACAGAAGCTGGTGGCTGTG-3' \\
& R: 5'-ACCCGCAAGTCTGTGCAATA-3' \\
IL-6 & F: 5'-AGTGAGGAACAAGCCAGAGC-3' \\
& R: 5'-GTGCCCATGCTACATTTGCC-3' \\
IL-1 $\beta$ & F: 5'-CTGAGCTCGCCAGTGAAATG-3' \\
& R: 5'-TCCATGGCCACAACAACTGA-3' \\
TNF- $\alpha$ & F: 5'-TCTTCTCGAACCCCGAGTGA-3' \\
& R: 5'-CTTGGTCTGGTAGGAGACGG-3' \\
Aggrecan & F: 5'-TCAGTGGTGACTTCACAGGC-3' \\
& R: 5'-TTCACCAACCGTAGGAGTGC-3' \\
Collagen & F: 5'-TGGCAAGCAAGGAGACAGAG-3' \\
type II & R: 5'-GACCTCTAGGGCCAGAAGGA-3' \\
$\beta$-actin & F: 5'-CGTGACATTAAGGAGAAGCTG-3' \\
& R: 5'-CTAGAAGCATTTGCGGTGGAC-3' \\
miR-140 & RT: 5'-GTCGTATCCAGTGCAGGGTCCGAG \\
& FTATTCGCACTGGATACGACCTACCA-3' \\
& R: 5'-GGCTAGTCAGTGGTTTTACCCT-3' \\
U6 & F: 5'-CGCTTCGGCAGCACATATAC-3' \\
& R: 5'-AAATATGGAACGCTTCACGA-3
\end{tabular}

F, forward; R, reverse.

5'-TCAAATACCATATTATGGTGTA-3' (the bold text indicates the mutation in the 3'-UTR of TLR4) and reverse 5'-AAACTTCTGCTGCAACTCTCATT-3'. The amplified cDNA fragments were subcloned into a psiCHECK-2 vector (Promega, Madison, WI, USA) at the XhoI and NotI sites downstream of the luciferase gene. A total of 293 cell lines were co-transfected with the luciferase reporter systems and miR-140 mimics or mimics NC as indicated in the figure legends. Luciferase activity was detected $24 \mathrm{~h}$ after transfection using the Dual-Luciferase reporter assay system (Promega) following the manufacturer's instructions. Data were normalized to Renilla luciferase activity.

Immunohistochemistry (IHC). Tissues were fixed in $10 \%$ neutral buffered formalin, embedded in paraffin, dewaxed in xylene and dehydrated in alcohol. Endogenous peroxidase activity was blocked by hydrogen peroxide pretreatment for $15 \mathrm{~min}$ using avidin/biotin blocking kit (ab64212; Abcam). Paraffin-embedded, formalin-fixed tissues were immunostained with rabbit polyclonal primary antibody against human TLR4 (1:500; cat. no. ab13556), IL-1 $\beta$ (1:300; cat. no. ab9722), IL-6 (1:300; cat. no. ab6672), TNF- $\alpha$ (1:400; cat. no. ab6671), aggrecan (1:500; cat. no. ab36861) and collagen type II (1:500; cat. no. ab34712) (all from Abcam) overnight at $4^{\circ} \mathrm{C}$. Rabbit IgG (cat. no. ab109489; Abcam) replaced the primary antibody as negative control (at an equal protein concentration) after washing the samples in 

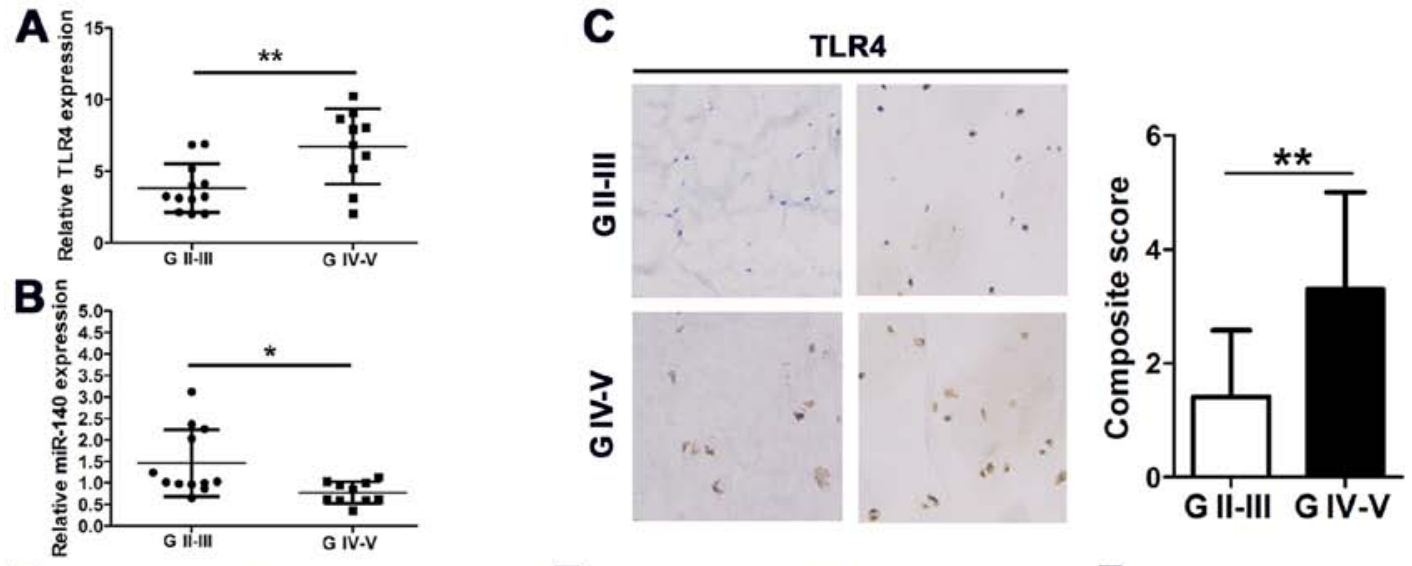

D

IL-6
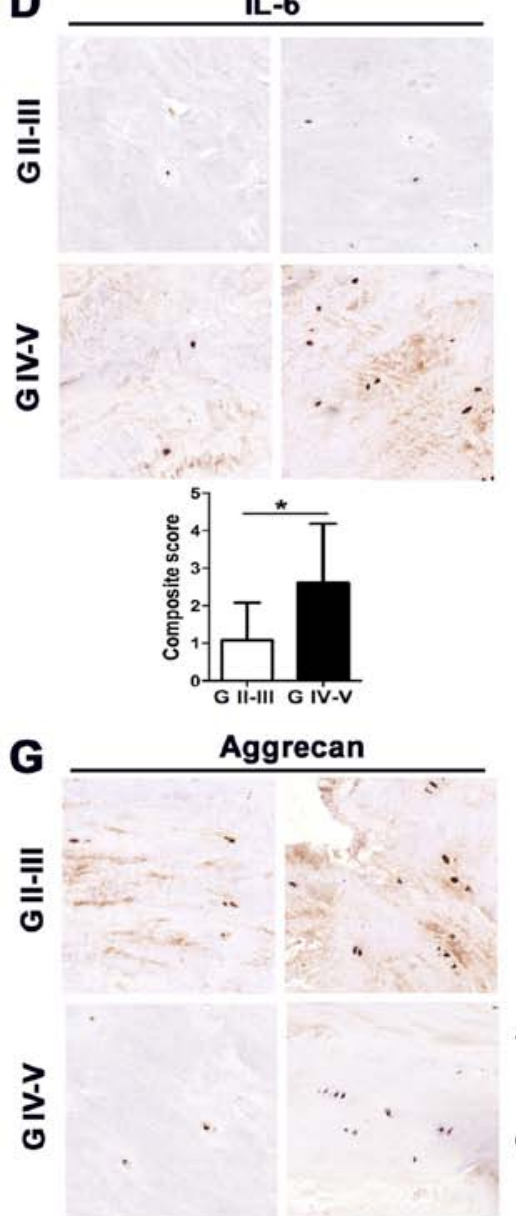

$\mathbf{E}$
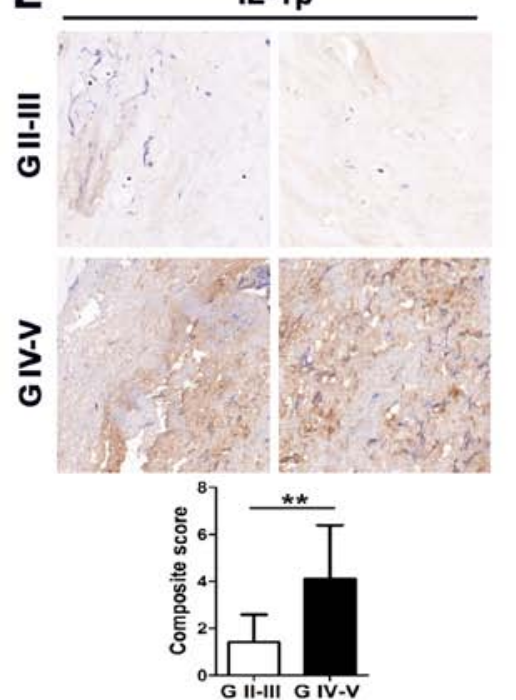

$\mathbf{F}$
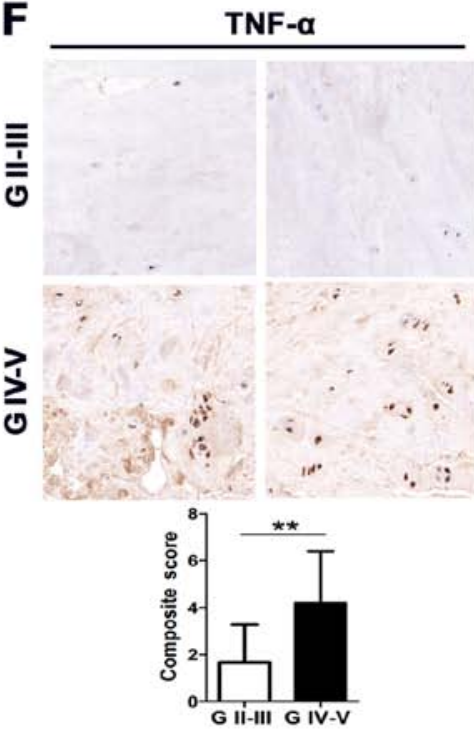
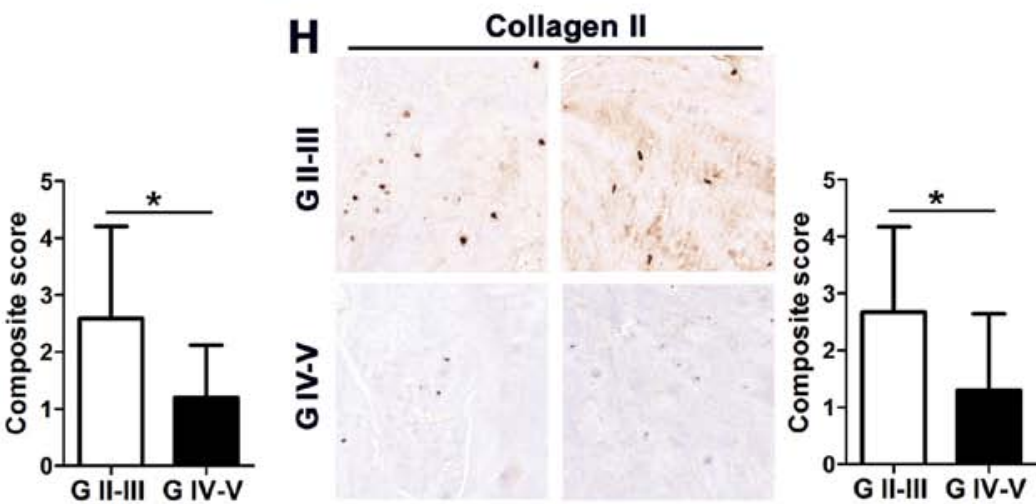

Figure 1. TLR4 is upregulated and miR-140 is downregulated in high-grade IVD degeneration tissues compared with low-grade tissues. (A and B) The expression of TLR4 and miR-140 in 12 IVD degeneration grade II-III tissues and 10 grade IV-V tissues was detected by qRT-PCR. (C-H) Representative images demonstrating TLR4, IL-6, IL-1 $\beta$, TNF- $\alpha$, aggrecan and collagen II expression (brown staining) and quantification by the multiplicative quickscore method as detected by IHC in 12 grade II-III and 10 grade IV-V tissues (magnification, $\mathrm{x} 200$ ). ${ }^{*} \mathrm{P}<0.05,{ }^{* *} \mathrm{P}<0.01$. G II-III, grade II-III; G IV-V grade IV-V.

phosphate-buffered saline (PBS). The second goat anti-rabbit HRP antibody (cat. no. ab80437; Abcam) was incubated for $30 \mathrm{~min}$ at room temperature. Then, the signal was amplified and visualized with diaminobenzidine-chromogen, followed by counterstaining with hematoxylin. TLR4, inflammation cytokines and ECM protein immunostaining were analyzed using intensity and distribution measurements as previously described $(55,56)$ and the staining intensity was determined as follows: 0 , no staining; 1 , weak; 2 , moderate and 3 , strong. The staining distribution was determined as the percentage of positive cells ( 0 , none; $1,<25 \% ; 2,25-75 \%$; and $3,75-100 \%$ ). The expression of TLR4, IL-1 $\beta$, IL-6, aggrecan and collagen II was evaluated by multiplying the intensity and distribution. Cells were further divided according to TLR4 expression into 'low' (TLR4 low) and 'high' (TLR4 high) groups, according to a cut-off point. The cut-off point for TLR4 expression was calculated using the X-tile software program (The Rimm Lab at Yale University (New Haven, CT, USA); http://www.tissuearray.org/rimmlab) as previously described (57). 
Table II. TLR4 staining and clinicopathological characteristics of 22 patients with IVD degeneration.

\begin{tabular}{lllll}
\hline & \multicolumn{2}{c}{ TLR4 } & & \\
\cline { 2 - 3 } Parameters & Low (\%) & High (\%) & Total & P-value \\
\hline Age (years) & & & & 0.096 \\
$\leq 45$ & $6(27.3)$ & $3(13.6)$ & 9 & \\
$>45$ & $4(18.2)$ & $9(40.9)$ & 13 & \\
Sex & & & & 0.145 \\
Female & $2(9.1)$ & $6(27.3)$ & 8 & \\
Male & $8(36.4)$ & $6(27.3)$ & 14 & \\
Body mass index & & & & 0.937 \\
$\leq 24 \mathrm{~kg} / \mathrm{m}^{2}$ & $4(18.2)$ & $5(22.7)$ & 9 & \\
$>24 \mathrm{~kg} / \mathrm{m}^{2}$ & $6(27.3)$ & $7(31.8)$ & 13 & \\
Disc level & & & & 0.190 \\
L3/4 & $5(22.7)$ & $3(13.6)$ & 8 & \\
L4/5 & $2(9.1)$ & $7(31.8)$ & 9 & \\
L5/S1 & $3(13.6)$ & $2(9.1)$ & 5 & \\
MRI grade & & & & $0.029^{a}$ \\
G (II-III) & $8(36.4)$ & $4(18.2)$ & 12 & \\
G (IV-V) & $2(9.1)$ & $8(36.4)$ & 10 & \\
\hline
\end{tabular}

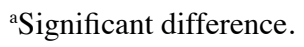

Cell immunofluorescence. For immunofluorescent staining, cells were first fixed in 4\% PFA for $10 \mathrm{~min}$ at room temperature, and then incubated overnight in anti-aggrecan (1:100; cat. no. ab36861) or anti-collagen II (1:100; cat. no. ab34712) antibody (all from Abcam), respectively. The following day, cells were labeled with the corresponding goat anti-rabbit IgG $\mathrm{H} \& \mathrm{~L}\left(\mathrm{Cy} 3^{\circledR}\right)$ preadsorbed ab6939 (Abcam) for $2 \mathrm{~h}$ at $37^{\circ} \mathrm{C}$, counterstained with DAPI and observed under an Olympus FluoView 2000 laser scanning confocal microscope (Olympus Corp., Tokyo, Japan).

Statistical analysis. Results are presented as the mean \pm standard deviation (SD). Data analysis was performed using SPSS version 13.0 (SPSS, Inc., Chicago, IL, USA). Student's t-test or one-way ANOVA was applied to test differences between the groups. Pearson's Chi-square test was used to analyze the relationship between the TLR4 expression and the clinicopathological features. $\mathrm{P}<0.05$ were considered to indicate a statistically significant result.

\section{Results}

Higher expression of TLR4 and inflammatory cytokines and lower expression of miR-140 and ECM in high-grade IVD degeneration tissues compared with low-grade tissue. The expression of TLR4 in the clinical characteristics of patients with high and low-grade IVD degeneration are detailed in Table II. There was no significant difference between the level of TLR4 expression and age, sex, body mass index and disc
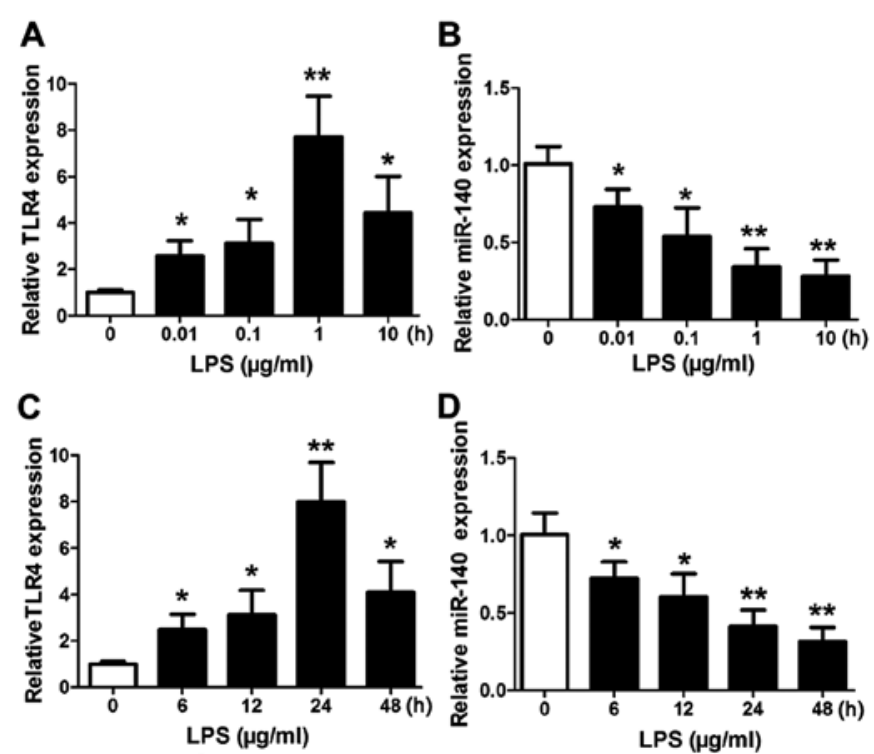

Figure 2. LPS induces the expression of TLR4 and downregulates miR-140 in human NP cells. qRT-PCR analysis of (A and C) TLR4 and (B and D) miR-140 expression in NP cells treated with LPS at the indicated concentrations for $24 \mathrm{~h}$ or LPS $(1 \mu \mathrm{g} / \mathrm{ml})$ for the indicated time-points. "P $<0.05$ and ${ }^{* * *} \mathrm{P}<0.01$.

level. However, TLR4 was expressed at a significantly higher level in the tissue of patients with high-grade IVD degeneration and at a significantly lower level in the tissue of patients with low-grade IVD degeneration. Relative expression of TLR4 and miR-140 was assessed by quantitative real-time PCR (qRT-PCR) in high- and low-grade (used as a relatively normal control) degenerative IVD tissue (Fig. 1A and B). TLR4 was found to be expressed at a higher level in grade IV-V tissues compared to grade II-III $(\mathrm{P}<0.01)$. In contrast, miR-140 was downregulated in grade IV-V tissues compared to grade II-III tissues $(\mathrm{P}<0.05)$. TLR4 was also detected by immunohistochemistry (IHC) in IVD degenerative tissues. Staining of 12 grade II-III tissues was less intense than that of 10 grade IV-V tissues indicating a high level of TLR4 in high-grade tissue (Fig. 1C). In addition, a higher level of the inflammatory cytokines IL-6, IL-1 $\beta$ and TNF- $\alpha$ was detected in high-grade tissues than in low-grade tissues (Fig. 1D-F). However, a lower level of aggrecan and collagen II expression was detected in high-grade tissues than in low-grade tissues (Fig. 1G and H). These results demonstrated that TLR4 and inflammatory cytokines were upregulated in the process of IVD tissue degeneration whereas miR-140 and ECM proteins were downregulated.

TLR4 is induced and miR-140 is downregulated in response to LPS stimulation. Subsequently, we assessed the expression of TLR4 and miR-140 in human NP cells stimulated with LPS. As expected, qRT-PCR analysis revealed that TLR4 expression was significantly increased in cells treated with a series of LPS concentrations (0.01-10 $\mu \mathrm{g} / \mathrm{ml})$ (Fig. 2A). The highest level of TLR4 expression was found in response to $1 \mu \mathrm{g} / \mathrm{ml}$ LPS ( $\mathrm{P}<0.01$ vs. the control). In contrast, the expression levels of miR-140 decreased significantly with increasing concentrations of LPS (Fig. 2B) with the lowest expression level in response to $10 \mu \mathrm{g} / \mathrm{ml}$ LPS ( $\mathrm{P}<0.01$ vs. the control). A time series of LPS $(1 \mu \mathrm{g} / \mathrm{ml})$ stimulation over $48 \mathrm{~h}$ revealed 

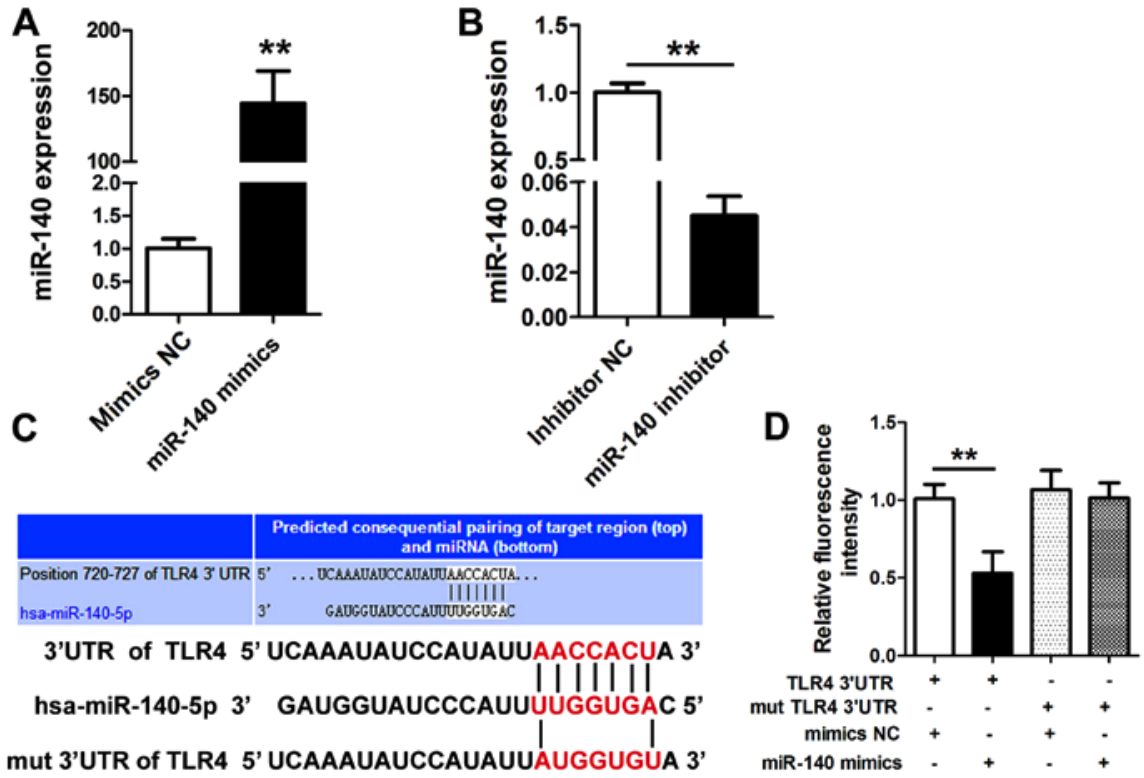

E
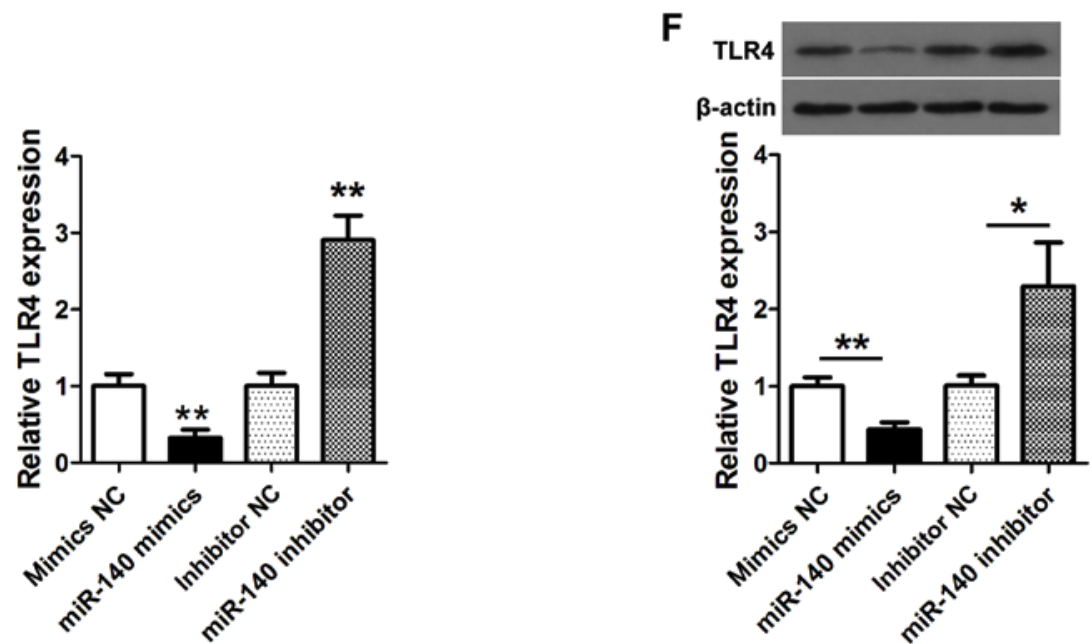

Figure 3. miR-140 is a target of TLR4. NP cells were transfected with miR-140 mimics, miR-140 mimics-negative control (mimics NC), miR-140 inhibitor, or miR-140 inhibitor-negative control (inhibitor NC) for $24 \mathrm{~h}$. (A and B) The expression of miR-140 was detected by qRT-PCR. (C) Putative miR-140 binding sites in the TLR4 3'-UTR. (D) A luciferase reporter plasmid containing wild-type or mutant TLR4 was co-transfected into 293 cells with miR-140 mimics or mimics NC. Luciferase activity was determined at $24 \mathrm{~h}$ after transfection using a dual-luciferase assay and shown as the relative luciferase activity normalized to Renilla activity. (E and F) qRT-PCR and western blot assessment of TLR4 expression in NP cells transfected with miR-140 mimics, mimics NC, miR-140 inhibitor and inhibitor NC. ${ }^{*} \mathrm{P}<0.05$ and ${ }^{* * *} \mathrm{P}<0.01$.

the highest levels of TLR4 expression were at $24 \mathrm{~h}(\mathrm{P}<0.01$ vs. the control), after which the levels began to decrease (Fig. 2C). Whereas, the expression of miR-140 was decreased (Fig. 2D) and continued to decrease with the lowest level at $48 \mathrm{~h}$ ( $\mathrm{P}<0.01 \mathrm{vs}$. the control). These results indicated that the presence of LPS induced TLR4 activation which reached a peak at a specific level. In contrast, the continued presence of LPS inhibited the expression of miR-140.

miR-140 directly regulates TLR4 by targeting its 3'-UTR. NP cells were transfected with miR-140 mimics, miR-140 mimicsnegative control (mimics NC), miR-140 inhibitor or miR-140 inhibitor-negative control (inhibitor NC) for $24 \mathrm{~h}$. The expression of miR-140 was detected by qRT-PCR (Fig. 3A and B) and confirmed the activity of the miR-140 mimic and miR-140 inhibitor. Fig. 3C displays the putative miR-140 binding site in the TLR4 3'-UTR. A luciferase reporter plasmid containing wild-type or mutant TLR4 was co-transfected into NP cells with miR-140 mimics or mimics NC. Luciferase activity was determined at $24 \mathrm{~h}$ after transfection using a dual-luciferase assay and demonstrated as the relative luciferase activity normalized to Renilla activity. A significantly lower luciferase activity was found with the miR-140 mimic then with the wildtype TLR4 3'-UTR ( $\mathrm{P}<0.01$; Fig. 3D). We then assessed the expression and protein levels of TLR4 in NP cells transfected with the miR-140 mimic and miR-140 inhibitor. TLR4 was significantly downregulated with the miR-140 mimic $(\mathrm{P}<0.01)$ while it was upregulated with the miR-140 inhibitor $(\mathrm{P}<0.01$; Fig. 3E). Similar results were obtained with western blotting $(\mathrm{P}<0.01$; Fig. 3F). The upregulation of TLR 4 in the absence of miR-140 or when the putative 3'-UTR binding site of miR-140 was mutated indicated that miR-140 may regulate $T L R 4$.

Overexpression of miR-140 inhibits the effect of TLR4 on IVD inflammation and degeneration induced by LPS stimulation. The influence of miR-140 on the expression of inflammation 

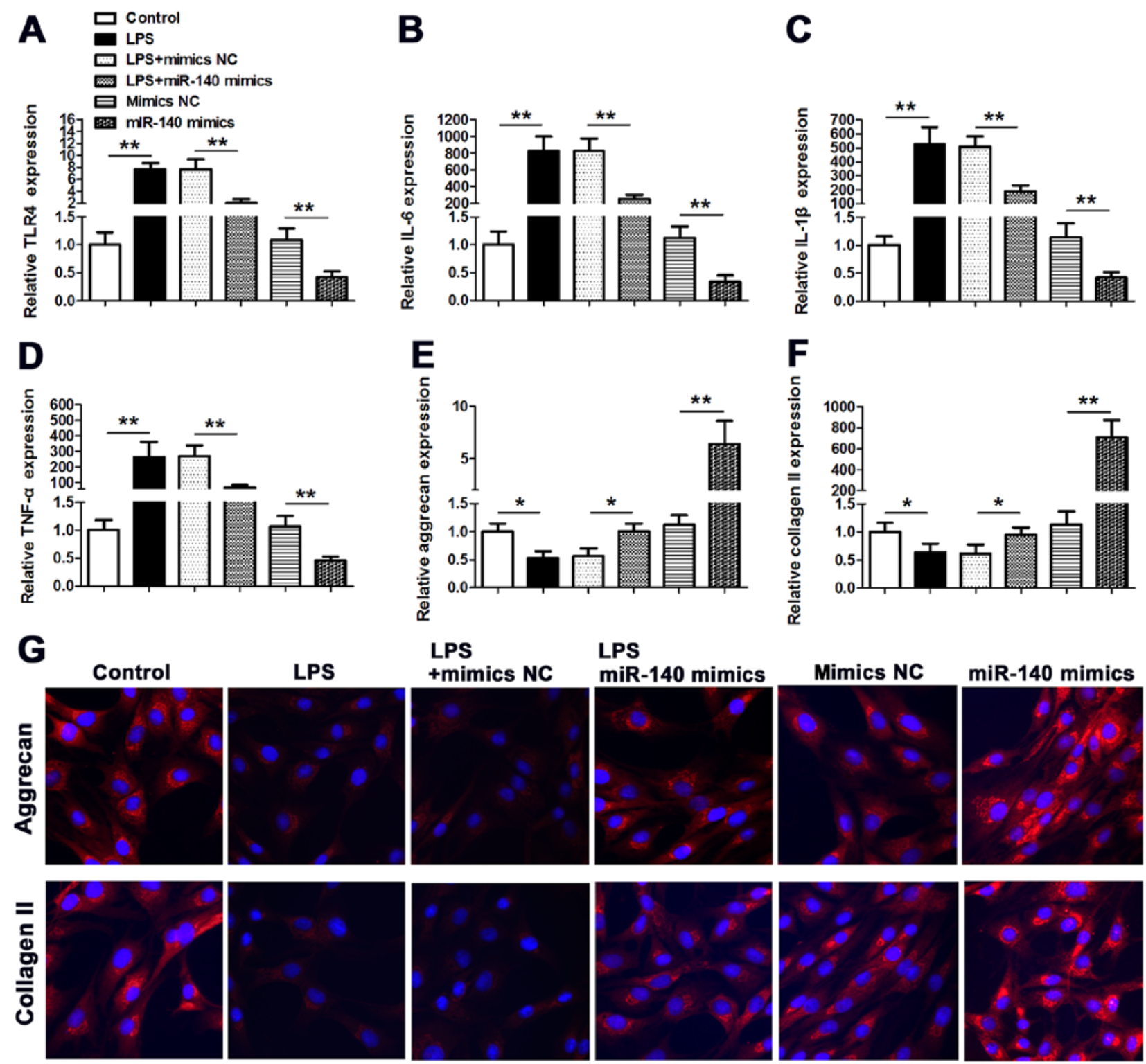

Figure 4. Effect of miR-140 on the expression of TLR4, IL-6, IL-1 $\beta$, TNF- $\alpha$, aggrecan and collagen II in NP cells with or without LPS. NP cells were stimulated with or without LPS and co-transfected with miR-140 mimics or mimics NC. Non-treated NP cells were used as a control. (A-F) qRT-PCR analysis of the expression of TLR4, IL-6, IL-1 $\beta$, TNF- $\alpha$, aggrecan and collagen II. (G) Aggrecan and collagen II were analyzed by immunofluorescence staining. Nuclei were stained with DAPI. Untransfected NP cells were used as a control. ${ }^{*} \mathrm{P}<0.05,{ }^{* *} \mathrm{P}<0.01$.

cytokines was assessed in NP cells stimulated with LPS. The expression of TLR4, interleukin (IL)-1 $\beta$, IL-6 and tumor necrosis factor (TNF)- $\alpha$ was significantly increased by LPS $(\mathrm{P}<0.01$ vs. the control), however, these increased levels of expression were lower in cells co-transfected with miR-140 mimic. Furthermore, without LPS treatment, the expression of TLR4, IL-1 $\beta$, IL-6 and TNF- $\alpha$ was also significantly lower in cells transfected with miR-140 mimic $(\mathrm{P}<0.01 \mathrm{vs}$. mimic $\mathrm{NC})$ (Fig. 4A-D). The expression levels of the ECM components aggrecan and collagen II were also assessed in LPS-stimulated NP cells (Fig. 4E and F). Aggrecan and collagen II were both downregulated in response to LPS stimulation ( $\mathrm{P}<0.05$ vs. control for both), whereas the expression of aggrecan and collagen II was reversed by co-transfecting cells with miR-140 mimic. In addition, without LPS stimulation, the aggrecan and collagen II expression were also significantly increased when the cells were transfected with miR-140 mimics $(\mathrm{P}<0.01$ vs. mimic NC). Aggrecan and collagen II expression was also visualized in NP cells by immunofluorescence staining (Fig. 4G). Nuclei were stained with DAPI and untransfected NP cells were used as a control. LPS stimulation clearly reduced the expression of aggrecan and collagen II in NP cells. However, in cells co-transfected with miR-140 mimic, aggrecan and collagen II levels were similar to levels in control cells under LPS treatment; however, without LPS stimulation, Collectively, these results imply that overexpression of miR-140 can inhibit the effect of TLR4 on IVD inflammation and degeneration induced by LPS stimulation.

Overexpression miR-140 modulates LPS-induced TLR4 expression and $N F-\kappa B$ activation. Finally, we assessed the TLR4 expression and NF- $\kappa \mathrm{B}$ activation in response to LPS stimulation in NP cells by measuring levels of $\mathrm{I} \kappa \mathrm{B} \alpha, \mathrm{p}-\mathrm{I} \kappa \mathrm{B} \alpha$, p65 and p-p65 protein expression by western blotting (Fig. 5). 


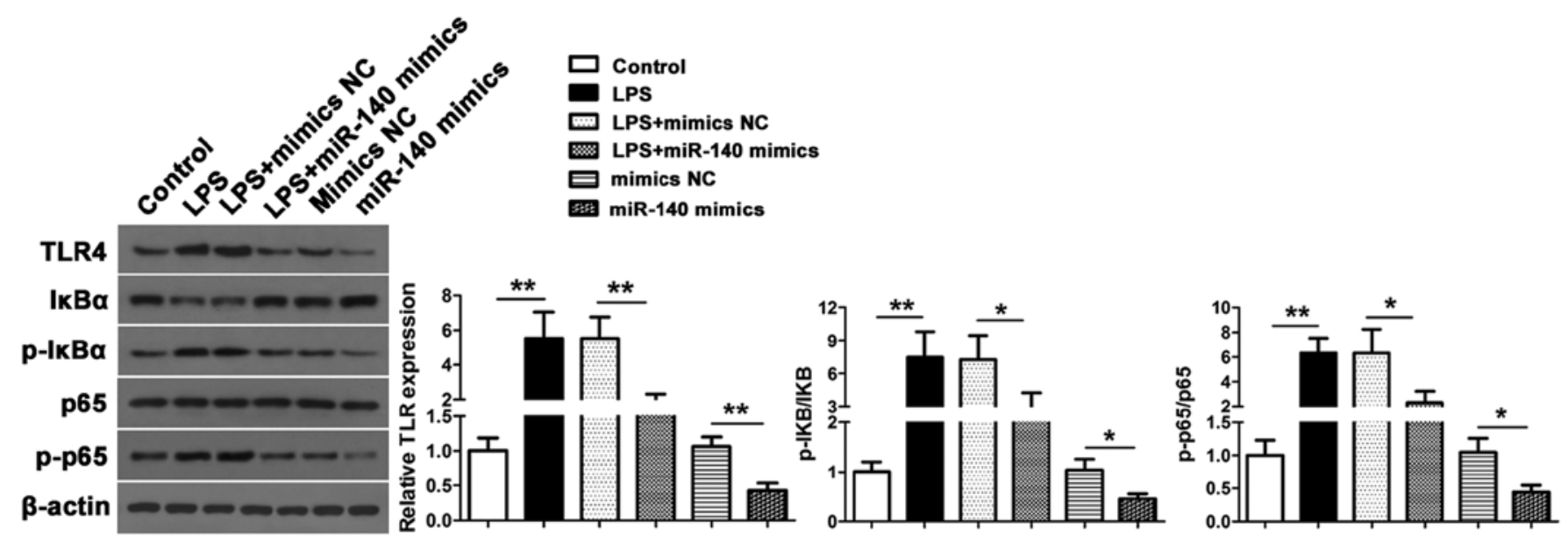

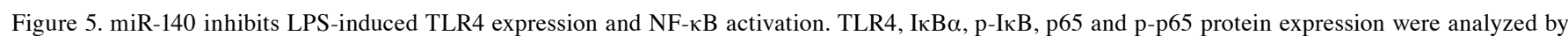
western blotting. ${ }^{*} \mathrm{P}<0.5,{ }^{* *} \mathrm{P}<0.01$.

Increased levels of TLR4, p-IKB $\alpha$ and p-p65 were found in NP cells after LPS stimulation $(\mathrm{P}<0.01$ vs. the control) but these levels were lower in miR-140 mimic co-transfected cells for TLR4 ( $\mathrm{P}<0.01$ vs. mimic NC), $\mathrm{p}-\mathrm{I} \kappa \mathrm{B} \alpha / \mathrm{I} \kappa \mathrm{B} \alpha(\mathrm{P}<0.05$ vs. mimic NC) and p-p65/p65 ( $\mathrm{P}<0.05$ vs. mimic NC). These results indicated that miR-140 inhibited LPS-induced TLR4 expression and NF- $\mathrm{KB}$ activation. Furthermore, without LPS stimulation, overexpression of miR-140 can also inhibit TLR4 expression and inhibit NF- $\mathrm{KB}$ activation.

\section{Discussion}

Multiple factors are reported to give rise to IVD degeneration and at present, many of these are irreversible, such as genetic disposition, aging or traumatic damage. Therefore, a method to alleviate the burden of this condition would be a suitable compromise. Collagen fibers are constantly challenged by direct radial pressure from the NP and cranial-caudal stretch from the separation of the two endplates $(5,9)$. Therefore, degenerated IVDs have a fibrous dehydrated nucleus with a bulging annulus fibrosus. In advanced stages of disc degeneration, inflammatory cytokines contribute to neurovascular in-growth and there is a progressive decrease in the expression of proteoglycans and collagen type II genes, which are important structural elements of the NP $(9,19)$. Furthermore, traumatic damage to the matrix leads to the accumulation of cytokines and several studies have implicated the role of TLRs in IVD degeneration $(31,35,36,58)$. The expression levels of TLR1/2/4/6 were found to be dependent on the degree of IVD degeneration (58).

In the present study, we have investigated the relationship between TLR4 and miR-140-5p in IVD degeneration. TLR4 expression was higher in high grades of IVD degeneration tissues than in low-grade IVD degeneration tissues whereas the opposite occurred with miR-140. Furthermore, the expression of TLR4 was associated with MRI grade in clinical characteristics of patients, but there was no significant difference in age, sex, body mass index and disc level. The expression of TNF- $\alpha$, IL-1 $\beta$ and IL- 6 was also higher in high-grade than in lowgrade IVD tissues. miR-140 was expressed at a lower level in high-grade IVD degeneration tissue compared to lower grade IVD degeneration. The expression of aggrecan and collagen II was also lower in high-grade than low-grade IVD degeneration tissues. TLR4 is a major receptor of LPS. LPS stimulation resulted in a significant increase in the expression of TLR4 and decrease in the expression of miR-140 in NP cells. Furthermore, LPS induced an increasing inflammation cytokine expression and decreasing aggrecan and collagen II expression. These results are consistent with previous studies that revealed that LPS inhibited aggrecan and collagen II synthesis in IVD and murine chondrocytes $(30,31)$. We mutated a putative binding site of miR-140 in 3'-UTR TLR4 and confirmed that the mutant gave a higher luciferase activity than the wild-type 3'-UTR TLR4 when the cells were transfected with miR-140, and indicated that miR-140 targeted TLR4; this result was consistent with the previous study by Li et al (49).

Overexpression of miR-140 inhibited the upregulation of TNF- $\alpha$, IL-1 $\beta$, IL-6, TLR4 expression and NF- $\kappa$ B activation and rescued the downregulation of aggrecan and collagen II induced by LPS stimulation. In a similar way, Li et al (44) revealed that miR-29a and miR-140 significantly reversed the effect of IL-1 $\beta$ pretreatment on chondrocytes, by influencing MMP13 and TIMP1 expression in both mRNA and protein levels, and subsequently affected the content of collagen II and aggrecan in chondrocytes. Karlsen et al (45) reported that overexpressing miR-140 increased the levels of proteins involved in the synthesis of hyaline ECM and reduced the levels of aggrecanases and other proteins that degraded the ECM, which could explain the extremely elevated levels of aggrecan and collagen II in response to miR-140 overexpression and the observation that the overexpression of miR-140 rescued the reduction of ECM expression induced by LPS stimulation in the present study.

Fu et al (39) assessed the expression of TLR4 in an LPS-induced kidney model. Compared with the control group, the phosphorylation levels of NF- $\kappa \mathrm{B}$ and I $\kappa \mathrm{B} \alpha$ were obviously increased in the LPS group. However, they revealed that the drug Tenuigenin dose-dependently inhibited LPS-induced TLR4 expression and NF- $\kappa B$ activation. NF- $\kappa B$ is usually located in the cytoplasm with I $\mathrm{B} \alpha$, but when it is stimulated by LPS it becomes detached from IкB and regulates cytokine production from the nucleus (59). TLR4 is a major receptor of LPS and regulates the NF-кB pathway (60). Recently, 
cordycepin, an extract from Cordyceps militaris, was found to suppress the LPS-induced activation of the NF- $\kappa \mathrm{B}$ pathway in NP cells (61). TLR4 activated the NF- $\kappa \mathrm{B}$ signaling pathway and induced the release of inflammatory cytokines production and $\mathrm{NF}-\kappa \mathrm{B}$ was reported to be responsible for the regulation of inflammatory cytokines production $(39,60,62)$.

We have found that TLR4 expression is increased in IVD degeneration and that the levels of TLR4 were negatively regulated by miR-140 with or without LPS stimulation. Li et al (49) reported in 2017 that miR-140 inhibited the expression of inflammation by downregulating the expression of TLR4. However, in the present study, we used an LPS-induced NP cell inflammation and degeneration model to activate TLR4 expression in vitro. In a previous study LPS was reported to increase inflammation expression and inhibit ECM expression in IVD in an in vitro and in vivo model (31). More functional in vivo model experiments are definitely needed in a further study to detect whether miR-140 can inhibit inflammation expression and ECM reduction through the downregulation of the expression of TLR4 which was induced by LPS stimulation. Furthermore, with or without LPS stimulation, miR-140 alleviated inflammation and degeneration by decreasing the level of cytokines and increasing the expression of aggrecan and collagen II by inhibiting TLR4 expression in NP cells. The present study may lead to a greater understanding of IVD degeneration and miRNAs may prove to be useful as markers or treatment agents in disease management.

\section{Acknowledgements}

Not applicable.

\section{Funding}

The present study was funded by the Health and Family Planning Commission of Changzhou Major Science and Technology Projects (ZD201504), the H-level Medical Talents Training Project (2016CZBJ033) and The Nineteenth Batch of Changzhou Science and Technology Plan (applied basic research) (CJ20160047).

\section{Availability of data and materials}

The datasets used during the present study are available from the corresponding author upon reasonable request.

\section{Authors' contributions}

DZ and NX conceived and designed the study. QZ, YW, YJ and SZ performed the experiments. QZ and YW wrote the paper. DZ and NX reviewed and edited the manuscript. All authors read and approved the manuscript and agree to be accountable for all aspects of the research in ensuring that the accuracy or integrity of any part of the work are appropriately investigated and resolved.

\section{Ethics approval and consent to participate}

All experimental protocols were approved by the Ethics Committee of Changzhou No. 2 People's Hospital.

\section{Patient consent for publication}

Not applicable.

\section{Competing interests}

The authors declare that they have no competing interests.

\section{References}

1. Zhang F, Zhao X, Shen H and Zhang C: Molecular mechanisms of cell death in intervertebral disc degeneration (Review). Int $\mathrm{J}$ Mol Med 37: 1439-1448, 2016.

2. Rannou F, Lee TS, Zhou RH, Chin J, Lotz JC, MayouxBenhamou MA, Barbet JP, Chevrot A and Shyy JY: Intervertebral disc degeneration: The role of the mitochondrial pathway in annulus fibrosus cell apoptosis induced by overload. Am J Pathol 164: 915-924, 2004

3. Kadow T, Sowa G, Vo N and Kang JD: Molecular basis of intervertebral disc degeneration and herniations: What are the important translational questions? Clin Orthop Relat Res 473: 1903-1912, 2015

4. Feng C, Liu H, Yang M, Zhang Y, Huang B and Zhou Y: Disc cell senescence in intervertebral disc degeneration: Causes and molecular pathways. Cell Cycle 15: 1674-1684, 2016.

5. Choi H, Johnson ZI and Risbud MV: Understanding nucleus pulposus cell phenotype: A prerequisite for stem cell based therapies to treat intervertebral disc degeneration. Curr Stem Cell Res Ther 10: 307-316, 2015.

6. Walter BA, Purmessur D, Moon A, Occhiogrosso J, Laudier DM, Hecht AC and Iatridis JC: Reduced tissue osmolarity increases TRPV4 expression and pro-inflammatory cytokines in intervertebral disc cells. Eur Cell Mater 32: 123-136, 2016.

7. Kepler CK, Ponnappan RK, Tannoury CA, Risbud MV and Anderson DG: The molecular basis of intervertebral disc degeneration. Spine J 13: 318-330, 2013.

8. Freemont AJ: The cellular pathobiology of the degenerate intervertebral disc and discogenic back pain. Rheumatology (Oxford) 48: 5-10, 2009.

9. Vergroesen PP, Kingma I, Emanuel KS, Hoogendoorn RJ, Welting TJ, van Royen BJ, van Dieën JH and Smit TH: Mechanics and biology in intervertebral disc degeneration: A vicious circle. Osteoarthritis Cartilage 23: 1057-1070, 2015.

10. Hayes AJ, Benjamin M and Ralphs JR: Extracellular matrix in development of the intervertebral disc. Matrix Biol 20: 107-121, 2001.

11. Roughley PJ, Alini M and Antoniou J: The role of proteoglycans in aging, degeneration and repair of the intervertebral disc. Biochem Soc Trans 30: 869-874, 2002.

12. Franceschi $\mathrm{C}$ and Bonafè $\mathrm{M}$ : Centenarians as a model for healthy aging. Biochem Soc Trans 31: 457-461, 2003.

13. Walker BF: The prevalence of low back pain in Australian adults. A systematic review of the literature from 1966-1998. Asia Pac J Public Health 11: 45-51, 1999.

14. Crockett MT, Kelly BS, van Baarsel S and Kavanagh EC: Modic type 1 vertebral endplate changes: Injury, inflammation, or infection? AJR Am J Roentgenol 209: 167-170, 2017.

15. Dudli S, Haschtmann D and Ferguson SJ: Fracture of the vertebral endplates, but not equienergetic impact load, promotes disc degeneration in vitro. J Orthop Res 30: 809-816, 2012.

16. Kanna RM, Shanmuganathan R, Rajagopalan VR, Natesan S, Muthuraja R, Cheung KMC, Chan D, Kao PYP, Yee A and Shetty AP: Prevalence, patterns, and genetic association analysis of modic vertebral endplate changes. Asian Spine J 11: 594-600, 2017.

17. Johnson ZI, Schoepflin ZR, Choi H, Shapiro IM and Risbud MV: Disc in flames: Roles of TNF- $\alpha$ and IL-1 $\beta$ in intervertebral disc degeneration. Eur Cell Mater 30: 104-116; discussion 116-117, 2015.

18. Le Maitre CL, Hoyland JA and Freemont AJ: Catabolic cytokine expression in degenerate and herniated human intervertebral discs: IL-1beta and TNFalpha expression profile. Arthritis Res Ther 9: R77, 2007.

19. Altun I: Cytokine profile in degenerated painful intervertebral disc: Variability with respect to duration of symptoms and type of disease. Spine J 16: 857-861, 2016. 
20. Wang J, Markova D, Anderson DG, Zheng Z, Shapiro IM and Risbud MV: TNF- $\alpha$ and IL-1 $\beta$ promote a disintegrin-like and metalloprotease with thrombospondin type I motif-5-mediated aggrecan degradation through syndecan-4 in intervertebral disc. J Biol Chem 286: 39738-39749, 2011.

21. Le Maitre CL, Freemont AJ and Hoyland JA: The role of interleukin-1 in the pathogenesis of human intervertebral disc degeneration. Arthritis Res Ther 7: R732-R745, 2005.

22. Xu F, Gao F, Liu Y, Wang Z, Zhuang X, Qu Z, Ma H, Liu Y, Fu C, Zhang Q, et al: Bioinformatics analysis of molecular mechanisms involved in intervertebral disc degeneration induced by TNF- $\alpha$ and IL-1ß. Mol Med Rep 13: 2925-2931, 2016.

23. Wang C, Yu X, Yan Y, Yang W, Zhang S, Xiang Y, Zhang J and Wang W: Tumor necrosis factor- $\alpha$ : A key contributor to intervertebral disc degeneration. Acta Biochim Biophys Sin (Shanghai) 49: $1-13,2017$.

24. Li X, Cheng S, Wu Y, Ying J, Wang C, Wen T, Bai X, Ji W, Wang D and Ruan D: Functional self-assembled peptide scaffold inhibits tumor necrosis factor-alpha-induced inflammation and apoptosis in nucleus pulposus cells by suppressing nuclear factor- $\kappa$ B signaling. J Biomed Mater Res A 106: 1082-1091 2018

25. Hu B, Shi C, Xu C, Cao P, Tian Y, Zhang Y, Deng L, Chen H and Yuan W: Heme oxygenase-1 attenuates IL-1 $\beta$ induced alteration of anabolic and catabolic activities in intervertebral disc degeneration. Sci Rep 6: 21190, 2016.

26. O'Neill LA and Dinarello CA: The IL-1 receptor/toll-like receptor superfamily: Crucial receptors for inflammation and host defense. Immunol Today 21: 206-209, 2000.

27. Janeway CA Jr and Medzhitov R: Innate immune recognition. Annu Rev Immunol 20: 197-216, 2002

28. Takeda K, Kaisho T and Akira S: Toll-like receptors. Annu Rev Immunol 21: 335-376, 2003.

29. Kim HA, Cho ML, Choi HY, Yoon CS, Jhun JY, Oh HJ and Kim HY: The catabolic pathway mediated by Toll-like receptors in human osteoarthritic chondrocytes. Arthritis Rheum 54 2152-2163, 2006

30. Bobacz K, Sunk IG, Hofstaetter JG, Amoyo L, Toma CD, Akira S, Weichhart T, Saemann M and Smolen JS: Toll-like receptors and chondrocytes: The lipopolysaccharide-induced decrease in cartilage matrix synthesis is dependent on the presence of toll-like receptor 4 and antagonized by bone morphogenetic protein 7. Arthritis Rheum 56: 1880-1893, 2007.

31. Rajan NE, Bloom O, Maidhof R, Stetson N, Sherry B, Levine M and Chahine NO: Toll-Like Receptor 4 (TLR4) expression and stimulation in a model of intervertebral disc inflammation and degeneration. Spine 38: 1343-1351, 2013.

32. Lu YC, Yeh WC and Ohashi PS: LPS/TLR4 signal transduction pathway. Cytokine 42: 145-151, 2008

33. Thompson JE, Phillips RJ, Erdjument-Bromage H, Tempst $\mathrm{P}$ and Ghosh S: I kappa B-beta regulates the persistent response in a biphasic activation of NF-kappa B. Cell 80: 573-582, 1995.

34. Lien E, Means TK, Heine H, Yoshimura A, Kusumoto S, Fukase K, Fenton MJ, Oikawa M, Qureshi N, Monks B, et al: Toll-like receptor 4 imparts ligand-specific recognition of bacterial lipopolysaccharide. J Clin Invest 105: 497-504, 2000.

35. Kim JS, Ellman MB, Yan D, An HS, Kc R, Li X, Chen D, Xiao G, Cs-Szabo G, Hoskin DW, et al: Lactoferricin mediates antiinflammatory and anti-catabolic effects via inhibition of IL-1 and LPS activity in the intervertebral disc. J Cell Physiol 228 1884-1896, 2013

36. Fang $\mathrm{F}$ and Jiang D: IL-1 $\beta /$ HMGB1 signalling promotes the inflammatory cytokines release via TLR signalling in human intervertebral disc cells. Biosci Rep 36: pii: e00379, 2016.

37. Chen B, Wang HT, Yu B, Zhang JD and Feng Y: Carthamin yellow inhibits matrix degradation and inflammation induced by LPS in the intervertebral disc via suppression of MAPK pathway activation. Exp Ther Med 14: 1614-1620, 2017.

38. Wan J, Shan Y, Fan Y, Fan C, Chen S, Sun J, Zhu L, Qin L, Yu M and Lin Z: NF- $\kappa$ B inhibition attenuates LPS-induced TLR4 activation in monocyte cells. Mol Med Rep 14: 4505-4510, 2016.

39. Fu H, Hu Z, Di X, Zhang Q, Zhou R and Du H: Tenuigenin exhibits protective effects against LPS-induced acute kidney injury via inhibiting TLR4/NF- $\kappa \mathrm{B}$ signaling pathway. Eur J Pharmacol 791: 229-234, 2016

40. Chen K and Rajewsky N: The evolution of gene regulation by transcription factors and microRNAs. Nat Rev Genet 8: 93-103, 2007.

41. Díaz-Prado S, Cicione C, Muiños-López E, Hermida-Gómez T, Oreiro N, Fernández-López C and Blanco FJ: Characterization of microRNA expression profiles in normal and osteoarthritic human chondrocytes. BMC Musculoskelet Disord 13: 144, 2012.

42. Miyaki S and Asahara H: Macro view of microRNA function in osteoarthritis. Nat Rev Rheumatol 8: 543-552, 2012.
43. Zhang $\mathrm{R}$, Ma J and Yao J: Molecular mechanisms of the cartilage-specific microRNA-140 in osteoarthritis. Inflamm Res 62: 871-877, 2013

44. Li X, Zhen Z, Tang G, Zheng C and Yang G: MiR-29a and miR-140 protect chondrocytes against the anti-proliferation and cell matrix signaling changes by IL-1 $\beta$. Mol Cells 39: 103-110, 2016.

45. Karlsen TA, de Souza GA, Ødegaard B, Engebretsen L and Brinchmann JE: microRNA-140 inhibits inflammation and stimulates chondrogenesis in a model of interleukin $1 \beta$-induced osteoarthritis. Mol Ther Nucleic Acids 5: e373, 2016.

46. Pando R, Even-Zohar N, Shtaif B, Edry L, Shomron N, Phillip M and Gat-Yablonski G: MicroRNAs in the growth plate are responsive to nutritional cues: Association between miR-140 and SIRT1. J Nutr Biochem 23: 1474-1481, 2012.

47. Conrad E, Polonio-Vallon T, Meister M, Matt S, Bitomsky N, Herbel C, Liebl M, Greiner V, Kriznik B, Schumacher S, et al: HIPK2 restricts SIRT1 activity upon severe DNA damage by a phosphorylation-controlled mechanism. Cell Death Differ 23: $110-122,2016$

48. Rodgers JT, Lerin C, Haas W, Gygi SP, Spiegelman BM and Puigserver P: Nutrient control of glucose homeostasis through a complex of PGC-1alpha and SIRT1. Nature 434: 113-118, 2005.

49. Li H, Guan SB, Lu Y and Wang F: MiR-140-5p inhibits synovial fibroblasts proliferation and inflammatory cytokines secretion through targeting TLR4. Biomed Pharmacother 96: 208-214, 2017.

50. Miyaki S, Nakasa T, Otsuki S, Grogan SP, Higashiyama R, Inoue A, Kato Y, Sato T, Lotz MK and Asahara H: MicroRNA-140 is expressed in differentiated human articular chondrocytes and modulates interleukin-1 responses. Arthritis Rheum 60: 2723-2730, 2009

51. Pfirrmann CW, Metzdorf A, Zanetti M, Hodler J and Boos N: Magnetic resonance classification of lumbar intervertebral disc degeneration. Spine 26: 1873-1878, 2001.

52. Lee JS, Jeong SW, Cho SW, Juhn JP and Kim KW: Relationship between initial telomere length, initial telomerase activity, age, and replicative capacity of nucleus pulposus chondrocytes in human intervertebral discs: What is a predictor of replicative potential? PLoS One 10: e0144177, 2015.

53. Chen C, Ridzon DA, Broomer AJ, Zhou Z, Lee DH, Nguyen JT, Barbisin M, Xu NL, Mahuvakar VR, Andersen MR, et al: Real-time quantification of microRNAs by stem-loop RT-PCR. Nucleic Acids Res 33: e179, 2005.

54. VanGuilder HD, Vrana KE and Freeman WM: Twenty-five years of quantitative PCR for gene expression analysis. Biotechniques 44: 619-626, 2008

55. Sive JI, Baird P, Jeziorsk M, Watkins A, Hoyland JA and Freemont AJ: Expression of chondrocyte markers by cells of normal and degenerate intervertebral discs. Mol Pathol 55: 91-97, 2002.

56. Zhu J, Ma J, Wang X, Ma T, Zhang S, Wang W, Zhou X and Shi J: High expression of PHGDH predicts poor prognosis in non-small cell lung cancer. Transl Oncol 9: 592-599, 2016.

57. Sun R, Wang X, Zhu H, Mei H, Wang W, Zhang S and Huang J: Prognostic value of LAMP3 and TP53 overexpression in benign and malignant gastrointestinal tissues. Oncotarget 5: 12398-12409, 2014.

58. Klawitter M, Hakozaki M, Kobayashi H, Krupkova O, Quero L, Ospelt C, Gay S, Hausmann O, Liebscher T, Meier U, et al: Expression and regulation of toll-like receptors (TLRs) in human intervertebral disc cells. Eur Spine J 23: 1878-1891, 2014.

59. Wang J, Guo C, Wei Z, He X, Kou J, Zhou E, Yang Z and Fu Y: Morin suppresses inflammatory cytokine expression by downregulation of nuclear factor- $\kappa \mathrm{B}$ and mitogen-activated protein kinase (MAPK) signaling pathways in lipopolysaccharidestimulated primary bovine mammary epithelial cells. J Dairy Sci 99: 3016-3022, 2016.

60. Fu Y, Hu X, Cao Y, Zhang Z and Zhang N: Saikosaponin a inhibits lipopolysaccharide-oxidative stress and inflammation in Human umbilical vein endothelial cells via preventing TLR4 translocation into lipid rafts. Free Radic Biol Med 89: 777-785, 2015.

61. Li Y, Li K, Mao L, Han X, Zhang K, Zhao C and Zhao J: Cordycepin inhibits LPS-induced inflammatory and matrix degradation in the intervertebral disc. PeerJ 4: e1992, 2016.

62. DiDonato JA, Mercurio F and Karin M: NF- $\kappa$ B and the link between inflammation and cancer. Immunol Rev 246: 379-400, 2012. 